\title{
Getting to What Matters: A Model of Perceptual Guidance
}

\author{
Anstein Gregersen
}

\begin{abstract}
This article outlines a model of perceptual guidance that aims at giving a child with congenital deafblindness perceptual access to the world, and in particular to the social world. It argues that we can closely interact with, and partially direct, a child's exploration without instructing the child into culturally similar ways of exploring. Perceptual guidance has two facets. The first is a bodily dimension where child and partner interact so that they create a joint bodily orientation to the world. The second is that partner and child show each other ways of organizing their overlapping perceptual field with arms and hands. This showing bears close resemblance to how we judge and discuss aesthetic matters. As in aesthetics, there is not a given correct procedure for exploring and judging, but an effort at creating community through showing each other how we experience the world. This gives the child access to the social world without purporting that this is the only correct way of exploring. The article ends with two practice examples of perceptual guidance, which additionally shows two ways of spatially organizing the perceptual field.
\end{abstract}

\section{Keywords}

Congenital deafblindness, tactile perception, communication, aesthetics, judgment. 


\section{Introduction}

How can a child with congenital deafblindness perceptually access and partake in his environment? ${ }^{1}$ In this article I propose a model of 'perceptual guidance' that suggests joint perception ${ }^{2}$ with the seeing and hearing partner as an access road to the environment and human culture. Building further on previous work (Gregersen 2020) in which I addressed some of the main objections against the idea of instructing persons with CDB's perceptual exploration, I here lie out an agency sensitive model of perceptual guidance. This method of guidance contains three features: it emphasizes joint perception; a culturally sensitive organization of perceptual fields, and a dynamic backand-forth mode of leading and exploring: (1) In close bodily alignment, the child and the partner jointly perceives the world. They do not just perceive the same object ${ }^{3}$, but also attune to each other's exploration. The partner enables this by showing the child possible ways of organizing their overlapping perceptual fields. (2) This perceptual organization occurs both in the selection of objects for perception, as well as in how to perceive them. This showing is culturally sensitive, enabling the child to understand a culture-like way of comprehending the object, while taking into concern the child's exploratory needs. (3) The child follows the partner because he trusts the partner to reveal the environment and culture in a manner that he can perceive and understand. Knowing that he also can lead and explore in his own way. The partner remains open and receptive to this agency on the child's part.

My predicament is that perceptual guiding is a continuous back-and-forth in whom leads and whom follows, partner and child together perceiving and interacting with the environment in search for community: A community and culture larger than the conversational dyad.

The model of perceptual guidance is developed specifically aiming at showing how the organization of the perceptual field is a communicative interaction. I start investigating the dynamic interaction between the guide and the guided by relying on the blind Canadian sociologist R. Michalko's first-person account of being in a dog guide team. From Michalko I borrow the strange, but potent idea of a world neither blind nor sighted that emerges out of the guiding. For Michalko this happens through a "Two in One" (Michalko 1999) interaction between the guide dog and the blind. I suggest we can fruitfully use these notions also in human guidance of children with CDB. I argue that both the "oneness" and the "twoness" results from communicative interactions, roughly corresponding to a whole-body form of communication and the explicit use of arms and hands in showing how to perceive.

The model is further outlined by suggesting parallels between the intellectual communicative activity of showing how to organize the perceptual field with the arms 
and hands and that of aesthetic judgments. For the philosopher Immanuel Kant aesthetic judgments were attempts at community building. In both aesthetics and in perceptual guiding there are diverse subjects that seek to build community although they have their own particular backgrounds, be it aesthetic taste or perceptual experience. In both cases, there are no rules or guarantees that the subjects will experience the same, but they strive towards it while accepting their differences. Therefore, both aesthetic judgments and perceptual guidance aim at agreement in perception while acknowledging and respecting that experiences hang together differently for people.

The article ends with a discussions of two recordings that, according to my model, illustrates successful perceptual guidance. Additionally, they show two different ways of practically organizing the perceptual field. In the first example, the partner and child encounter a singular and stable object. The partner is concerned with the order of appearance, first showing the whole object, before moving on to the parts. In the second, and more innovative, example the partner and child joins two other children playing in what is a complex and fast changing situation. The partner organizes the perceptual field by a whole-body orientation that aligns the child and partner with the other children. This creates a space that the child potentially can perceive. Further, the partner's arms do not show all the components of the play but stretches out as orientation lines into the spatial core of the play. The child exploits this organization to engage in the play.

\section{The model of perceptual guidance: A world neither blind nor sighted and bodily interaction.}

Perceptual guidance implies that the partner, somehow, directs the child's perception. This goes against the grain of much of deafblindstudies (see Gregersen 2020 for further discussion) that worries that directing a child with CDB's perceptual exploration amounts to instructions into culturally expected forms of exploration (Nafstad and Rødbroe 2007). There are several good reasons for such a worry: Children with CDB often use other tactile and sensory resources than seeing and hearing peers, the chin might explore a carrot, and the mouth a cell phone. The significance of a touch might also differ from how a seeing and hearing peer perceives it. Children with CDB often uses other criteria than an encultured seeing and hearing peer in comparing and categorizing objects, events etc.. There is a risk that the way a child with CDB perceptually explore the world will not be acknowledged (Souriau and Brede, 2008). And that the cultural-similar way of perceiving that the partner instructs into might not fit the child's need. Shortly put, due to the diversity in experiences that CDB represents, and internal variation within the group of persons with $\mathrm{CDB}$, instructions into a mainstream way of exploring is problematic. 
While taking these concerns seriously I still think some form of directing, though not physical enforcement, of perception is beneficial for giving the child access to the environment for at least three reasons: The first reason concerns perceptual economy. Tactile perception is both very time consuming and mentally taxing. With perceptual guidance, a child can off-load perceptual work to the partner so that it can concentrate on perceiving the most important parts of the environment. The second reason concerns access. The time demandingness of tactile perception makes many fast-changing events, e.g. social interactions, not accessible without some guidance based on sight. The third reason also concerns access. Perceptual guidance can offer the child possibilities of understanding how his peers and the rest of culture encounters the environment (see also Gregersen 2020). This can enrich him in kinds of experiences. Moreover, conditions for social comprehension and interaction improves, and in the end the child's selfunderstanding off his position in the social world as congenital deafblind.

The use of seeing and hearing dog guides is common in the blind community. I want to look at one sophisticated account of these interactions for resources renderable into the domain of human guiding of children with CDB. In The Two in One Michalko offers us a combination of sociological theory and first-person experiences from longterm interactions with guide dogs. These personal experiences lend credibility to his understanding of guiding, and one of his main concerns is the dynamic in leading and following.

Guide dogs are of course important for mobility, but for Michalko the dynamic interaction with guide dogs gives him also perceptual access - yet, again - to the world. Though it isn't exactly the same world he was familiar with before his blindness. Michalko's first experience is with the dog Leo:

Leo did not "perfect" my sense of touch by making it more sensitive. I was not able, harness in hand, to touch things I had not touched before or to feel things more perfectly. Instead, my grasp on the harness handle brought the tactile character of the world closer to me and I thereby became more "in touch" with my sense of touch. Rather than feeling my way around the world, as I did with the white cane, my brief experience with Leo gave me an awareness of how the world expressed itself through tacticity. I was not so much relying on my sense of touch to get around as I was re-arranging my sensual experience and subsequent understanding. (1999, p.32)

A blind person needs a guide who will not merely move her through the world but also bring that world to her and take her to it. Guides bring blindness and sightedness together in a world which is neither "blind" nor "sighted" but within which they both appear and live. (1999, p.32) 
The guide dog brings Michalko closer to the tactile character of the world and teaches Michalko about how the world expresses itself through tacticity. Remark though that he does not equate that world with what he can touch. The world the guide brings Michalko into is larger than what he can touch. It is a world where the environment outside of Michalko's tactile field expresses itself through tacticity thanks to the guide`s sight. This idea of a world neither blind nor sighted is potent because it points towards a way of approaching the world that allows a child with CDB to experience and partake in a world that is close in some respects to the one seeing and hearing children live in, but it also the child's home-ground shaped through tactile encounters.

How is it that the interaction with the guide dogs brings forth this world neither blind nor sighted? Michalko describes this interaction as "together alone" and "two in one". What these descriptions points at is the possibility of using the guide`s sight and hearing while keeping agency and autonomy. This is what perceptual guiding must achieve both in theory and in practice. We start with the one-ness. Michalko relates the experience of first holding Leo in a harness:

I experienced a sudden and surprising sense of security. I was holding the harness handle lightly in my hand as the trainer instructed me to do, but in spite of the lightness of my grasp, I felt a sense of stability that seemed to come from the firmness and strength of Leo's shoulders. I also experienced a sense of distance, an expansion of my immediate environment. It seemed as though my "sense of touch" was enhanced by Leo and his harness. I could "feel" further than I ever had before. Harness and Leo in hand, I felt my tactile sense replacing my sense of sight as the "distance sense. (Michalko 1999, p.25/26)

Notice Leo's impact: Michalko now feels secure and experiences a sense of distance and expansion of his immediate environment. Later Michalko says about his long-term guide Smokie that: "Smokie has returned my body to me." (1999, p.122). I suggest that there are two stages to this bodily connection between Michalko and Smokie. The first is that Michalko yet again experiences his body as a perceiving body, a sense-making body, that intentionally relates to the world. How can this happen?

Smokie provides Michalko perceptual stability, and stability is a pre-condition for access. Smokie is in tactile reach for Michalko. Moreover, the dog's perceptual system reaches beyond Michalko`s. Smokie is oriented to the world through sight, and he is a trained dog that knows how to communicate to Michalko the significant aspects of the world that a guide dog is supposed to communicate. No doubt, Smokie has a personality of his own, and through interactions with Michalko he communicates more than his 
training taught him. Nevertheless, through perceiving, communicating and simply being bodily oriented to the world together with Michalko, Smokie structures the world so that when it enters Michalko`s perceptual field through the harness it is stabilized, and has pattern that is perceptible through touch. Michalko`s perceiving body is yet again meeting a world that it can be intentionally oriented to, as a world that makes sense through the guide dog's actions.

The second stage comes when Michalko and Smokie continuously interact. Their bodily interactions are "Something dynamic and fluid that flows from one partner to the other and back again" (1999, p.7), "a fluid relation that does not apply when leader and follower are understood as static and completely separable entities" (1999, p.185). Michalko will say about his long-term guide dog Smokie that they are extensions of one another (1999, p.5).4

This tactile interaction between Michalko and the guide dog - where their bodies align and jointly orient to the world - is an important part of how Michalko accesses the world. My practical recommendation for perceptual guidance is close in kind: It is a prolonged tactile contact where partner and child bodily align and jointly orient to the world. The guiding takes-up significant amounts of time, and for a full account we therefore need a description of what happens when two bodies interact closely in this aligned way (for a longer discussion bodily alignment see Gregersen 2018).

Why does this one-ness occur? Can it transfer to partner and child interactions? Research in social psychology provide one explanation. Soliman et al. (2015) suggests that one consequence of joint action is that we get entangled with our partners. Consider dancing. Soliman et al. asks if while dancing "you adjust your body schema, that is, how you move and perceive your own body, to take into account your partner's actions? Furthermore, does that adjustment continue after you leave the dance floor?" (2015, p. 873). They answer these questions positively arguing that joint actions construct a joint body schema (JBS) among the dyadic partners:

We show through converging behavioral methods that joint action induces the emergence of what we term a JBS between members of the collaborating dyad. The JBS is a temporally extended spatial representation referencing the dyadic partners' body states, and it affects behavior even after the joint task is completed. (Soliman et al. 2015, p. 883).

This provides one possible explanation of the "oneness". Moreover, it explains how perceptual guiding at a bodily level would bring a child with CDB to the world "neither blind nor sighted" through his partner's body schema that has emerged out of sight and hearing. 
This brings us to the "two-ness" of Michalko's "two in one". "Two in one" is a phrase frequently employed by the philosopher Hannah Arendt (see e.g. Arendt 1985) to describe that in thinking by oneself there is an internal dialog between (at least) two parts. For our purposes, the two-ness must imply that the child autonomously experiences, thinks and communicates. For Michalko the two-ness stems from the fact that Smokie is a dog and Michalko is a man. Therefore, while they are bodily as one, they are also separate as man and animal:

Thus Smokie and I live and work alone together in our society, he as domesticated nature and I as an example of nature "gone wrong." Smokie is a reminder of society's power over nature, while I remind society of nature's power over it. We are at home in our society and are familiar with its landscape and customs, though Smokie's familiarity comes from the point of view of his domesticated nature and mine comes from the point of view of blindness. We remain foreigners in our homeland despite our familiarity with it. We see our homeland from the point of view of "estranged familiarity". (1999, p.112)

Michalko sees the world "neither blind nor sighted" as emerging from a “contrapuntal 'awareness of simultaneous dimensions'..." (1999, p. 109-110), those of sight and blindness. These dimensions are realized in the interaction with the sighted guide dog. The child with CDB, however, is not exiled from an original familiarity with culture. The task is not a return to a homeland, but creation of a homeland. This should be through a contrapuntal awareness that will reveal significant features of the sighted world while simultaneously letting the child discover his difference from it. For such a contrapuntal awareness, the child will need a guide into human culture. This sighted guide needs a different cultural understanding than what, with all respects intended, a dog can provide.

How can we construe the two-ness in guiding into culture? Perceptual guiding is a deliberate communicative activity where the partner shows the child ways of organizing his perceptual field. We can take a lead from aesthetics about organization of the perceptual field and community. I will concentrate on the arms and hands in this organization, noting that other body parts can be used as well.

\section{The model of perceptual guidance: Judgment, communication and organizing perception.}

For approaching the explicit organization of the perceptual field with arms and hands, we briefly turn to a distinction between two types of judgment found in 
Immanuel Kant's Critique of Judgment ${ }^{5}$. For elaborating on the distinction, I will consider two judgments about the Arab castle Alhambra, located in the Andalusian city Granada. P1 ("P1" standing for "proposition6 1") says:

"The Alhambra complex is an Arab citadel".

In P1, the judgment subsumes the particular entity, Alhambra, under the concept 'Arab citadel'. P1 is what Kant called a "determinative judgment": it determines what the particular entity is by subsuming it under a concept. ${ }^{7}$ Kant's idea is that we for the most part have rules, or schemas, for determining what a particular entity is by subsuming it under a concept. We have for instance rules for what counts as "Arab" and what counts as "citadel". Knowing these rules, Alhambra becomes an example of the rules. The presumption behind a determinative judgment is that we know the schema for subsuming a particular object under the general concept. In judging Alhambra to be an Arab citadel, one judges that some of the manifold of the castle is the significant features, while other features reside to the background. There are plenty of possible determinative judgments to be made about Alhambra: "Alhambra is a tourist machine"; "Alhambra is made of red stones" and so on. The determinative judgment brings some features of the manifold to the fore, while others withdraw.

Many children with CDB will not easily acquire the same schemas as their seeing and hearing peers. One reason for this is that it is difficult for a child with CDB to know what features of the manifold to take as the significant ones. Simply attaching a sign to a perceived object will not necessarily remedy this neither, since it is possible that the child will perceive other features of the object than intended by the conversation partner. This consideration, someone might argue, points towards simply instructing the child how to subsume different objects under different concepts.

In order to see the shortcomings with such a strategy, recall that Michalko described the world he and Smokie constituted together as a "world neither blind nor sighted" (1999, p.32). In other words, even a person with acquired blindness describes his experiences as hanging together differently from those reported by seeing people. In line with this recognition of diversity, I think we should operate with the presumption that the way a child with $\mathrm{CDB}$ experiences is different from the seeing and hearing partners. Simply insisting through instructions, then, that the child with CDB subsumes an object under the same concept as seeing and hearing peers does not respect sufficiently that a child with CDB's experiences hangs together differently.

Instead of simply insisting on certain ways of encountering objects, the child and partner must find perceptual organizations so that "a world neither blind nor sighted" emerges. In other words, the joint perceiving of the guide and the guided has a creative 
dimension. This is the first of two reasons for why we should turn to aesthetics. The second is that aesthetic sensibilities are important in culture, learning and development (Trevarthen 2011). They are at the same time inextricably linked to subjectivity, and the meeting of subjectivities in the public realm. The world "neither blind nor sighted" that guide and guided appear in resembles this in that there is a common world where two subjectivities meets and at the same time create that world.

For Kant reflective and not determinative judgments tie to the aesthetic. Reflective judgments are in use when determination is not possible or, as in the case of the aesthetic, not really the goal:

To reflect (or consider [überlegen]) is to hold given presentations up to, and compare them with, either other presentations or one's cognitive power [itself], in reference to a concept that this [comparison] makes possible. The reflective power of judgment is the one we call the power of judging [Berurteilung] (facultas diiudicandi). (Kant, 1987, p.211)

Kant thus thinks that the reflective power of judgment can do two kinds of comparisons. The first is comparison between presentations, or perceptions, where we perceive an object, but do not have a concept for determining the object. In this case, we compare perceptions in order to create a new concept. In haptic perception, for instance, a common exploratory procedure by a person with blindness is to follow edges for perceiving the contour of an object (see Heller and Gentaz 2014). The perceiver through a time-sequence compare the different perceptions of the edge in following it. In addition, the perceiver must often compare the perception of the object to his own perceptual system for perceiving hardness, temperature and so on. When encountering new kinds of objects, or new contexts the object appears in, we must create a concept of what we perceive. So far, we have described the creation of new concepts as an individual process, but it is of course also a social process. One dimension of the "world neither blind nor sighted" is that how the concepts captures objects will often be somewhat different from how the concepts work among seeing and hearing peers.

Let us return to the quote from the Critique of Judgment and the second kind of comparisons that is possible through reflection. For Kant aesthetic judgments are the primary example of the comparison between the presentation of an object and our cognitive power itself. Let us take a short internet trip back to Andalucía. On the English version of the Granada Gity Council Official Tourist Information Web Page we find a section called "What to do?" After clicking that banner, the "What to visit" takes us directly to a page called "What to see". This page, however, does not provide an inventory of what there is to see in Granada in the sense of "things that are visible". Such a list 
would go on forever, and not be of much value to anyone. In the same way, it would of course be possible for the partner in perceptual guidance to attempt showing the child with CDB the whole inventory of objects in a given space. This obviously being a futile project the partner must judge some objects as more important than other objects. Shortly put, the Tourist Information Web Page and the perceptual guide must focus on what is significant, on what matters. Returning to Andalucía and Granada, which is a city of many vistas, the web page writes about Granada's most famous tourist attraction that P2:

“The Alhambra complex is the world's most spectacular Arab citadel...".

Recall that P1 expressed the judgment: "The Alhambra complex is an Arab citadel", so at first glance, it seems the only difference between P1 and P2 is the added "the world's most spectacular". However, "spectacular" is not only about the castle, it is also about the one who visually perceives the citadel. In P2, there are three elements: 1 ) an "I" that makes a judgement based on the subject's own aesthetic experience of 2) the object and 3) the judgment addresses a community as-if this judgment holds for the whole community. Or, the whole world in this case. Aesthetic judgments, according to Kant, are reflective because they reflect on the relationship between the judging subject, the object and the community of fellow judgers. The judgment in P2 that "Alhambra...is spectacular" does not hold for a community the same way that P1 does since P2 voices the subject's own feelings of what is spectacular. Since there is a diversity among people in what they deem spectacular; beautiful; ugly; tasteful; distasteful and so on, there are for Kant no rules or standards that we can simply apply in determining what aesthetic evaluation is correct. When we do not know what the concept to subsume the particular object under, we reflectively judge in order to create a new concept, a new universal. In aesthetic judgments Kant argues, the goal is also universality: a universal aesthetic agreement among fellow judgers.

P2 voices a universal claim that the tourists should look at Alahambra because it is spectacular. At the same time, the tourist information web page makes a claim about how we should see Alahambra: We ought to see it is as spectacular. I am going to call this an "exemplar judgment". It is an individual judgment giving an exemplar of how others should organize their perception of an object. So, the judgment is not an example of the rule governing when to apply "spectacular", but an exemplar of how to perceive Alahambra. The goal of the universal voice in P2 is therefore agreement with fellow judges, or community creation.

In aesthetic judgment, then, Kant thinks subjects attempt to create community. The aesthetic judgment aims at a community of sensing humans (sensus communis) in 
the movement from the subject through a judgment of an object that seeks agreement. The goal is community creation among perceivers with varied aesthetic sensibilities: attuning in the common orientation to the world.

In the act of comparison, reflective judgment, as opposed to determinative judgment, does not work mechanically or schematically, but artistically and creatively (Kant, 1987, p. 214). Reflective judgment is situation and context dependent and cannot rely on pre-given schemas. These are then open, or receptive, judgments. They answer to objects in the world. Searching for new concepts or agreements between perceivers.

Reflective judgment is an apt description of the basis of perceptual guidance: The goal is to make access to culture and human interactions possible through organizing our perceptual fields. Working on the premise that the child is a cognitive and social creature the partner offers the child exemplars of how to organize the perceptual field. These demonstrations can be accepted, rejected or modified in a back-and-forth between the partner and child. The organization must be artistic or creative in the constitution of a social space between an individual with a highly distinctive lifetrajectory and a partner that has participated in culture through sight and hearing. In that the comparisons depart, so to speak, from the subjective realm we must accept the authority of the child in how he wants to organize his perceptual field. At the same time, he must have access to our way of organizing it in order to comprehend us.

Summing up, the model of perceptual guidance is dyadic, but not a conversational dyad. The partner and child bodily align in encountering the world. On the bases of this bodily alignment, they can share spatial perspective. Within the common perceptual field, they show each other possible ways of perceiving the environment, or possible ways of organizing their overlapping perceptual field. This organization is a kind of aesthetic activity. It is creative, not rule bound, and aims at establishing a community of experiencers: diverse subjects experiencing the world in common and commonly. The creativity of the partner is showing the child organizations of the perceptual field that attunes to how seeing and hearing culture would experience it. However, the suggested perceptual organization by the partner also attunes to how the child potentially can experience the environment. The child can exploit the partner's suggestions by creatively integrating them into his own way of experiencing. Through this joint creative sense-making process a world neither blind nor sighted emerges that enables the child to participate in the human environment

\section{Practice examples.}

This last part discusses two video recordings of guiding that illustrates the model of perceptual guiding. Additionally, the recordings show two different ways of spatially 
organizing the perceptual field, and they are as such a first contribution to a taxonomy of techniques in perceptual guiding. Both recordings are of my son, Kasper, when he was around three years old. He was born deaf and blind with no-light perception and was most likely completely deaf from birth. By his first year he had bi-lateral CI and enjoyed sounds and understood quite a bit of verbal communication. In addition to using conventional signs, he also spoke a few words. Due to low muscle tone and ataxia, he was at the age of 3 years still carried forward facing in a baby carrier. This secured close physical interactions with his partners that resembles the tactile connection between Michalko and the guide dog, what I have previously called "body-with-body" (Gregersen 2018).

Recording I.

In the first recording ${ }^{8}$ Kasper hangs forward facing in a baby carrier on his partner. They are walking in the hallway of a Snoezelen multisensory centre. The partner brings Kasper to a wall-mounted box:

Part (A) from 0.00 to 0.02: The recording starts when the partner and Kasper have reached a rectangular sound box mounted to a wall at the height of a smaller child. The box has an oblong shape; it is wide and low. Horizontally arranged after each other are eight large round buttons with different colours that give off various animal sounds when pressed. At first standing, the partner presses a yellow button with pig-sound.

Entering the hallway seeing and hearing children would swiftly see the whole room, or "manifold" as Jonas (1954, p. 507.) called it. Seeing and hearing children would also see the sound box and the different colours of the buttons.

For Kasper, entering the hallway, the sound box is outside his perceptual field and the partner has decided that the right action is not to let him discover the hallway on his own. Furthermore, the partner thinks there is an important object in the room that they should engage together. One aspect of this guiding entails perceptual economy. Kasper is off-loaded from having to investigate the whole room himself; this saves him both time and energy. It allows him in this situation to perceive an object and play with it for a short while before he is scheduled for swimming.

Another aspect of this guiding is cultural sensitivity. The partner brings Kasper to an object made for seeing and hearing children. This is a community building effort geared towards sharing experiences: a sharing between Kasper and the partner and indirectly with other seeing and hearing children by giving him access to the kinds of experiences they have. 
One might object to this guiding that Kasper is not free to figure out himself what he wants to engage. Yet, the partner addresses this by means of an open communication that invites him to join her in engaging the object. First, she does this by pushing the button with the pig-sound as to demonstrate what the box can do. Secondly, she asks Kasper to share her interest in the box by showing him the object:

Part (B) from 0.03 to 0.52: The partner leads Kasper's hands horizontally over the top edge of the box, while their hands together push some of the buttons. 10 seconds into the recording the partner positions herself differently and kneels. They together feel the top edge again with both hands: their left hands follow the edge leftwards, their right hands rightwards. The hands move down the left and the right side almost simultaneously, before following the bottom edge so that the hands meet in the middle. They have now perceived the four most significant edges of the box. The partner then supports Kasper's arms, so he again pushes some of the buttons while she verbally says, "It is to be played".

By holding her right arm under the elbow of the Kasper's right hand, she supports his arms that are weak, due to low muscle tonus. Kasper presses the buttons on his own. The partner then says: "That was fun. Shall I do that too?" and she pushes some buttons as well. At 00.52 Kasper moves his hands without support. He explores the top edge again, left hand starting from left side and right hand from the right side almost meeting right in front of his body. His arms then move apart by the left hand following the bottom edge leftwards and the right hand rightwards.

The invitation to engage with the object continues here by showing Kasper the outlines of the object. For approximately the first thirty seconds of the recording Kasper is in "attentive modus" to what the partner is doing. Then he starts exploring himself. This part of the recording illustrates the back-and-forth dynamic in guiding. To rebut the objection that Kasper is not sufficiently free to explore on his own, it is important that Kasper is in "attentive modus" in the beginning. For those who know him it is clear that he is paying attention and waits for the partner to show him something. The showing of the object is also rather quick, and then the partner lets him respond to whether he is interested in the object or not by letting him perceive it. After his attentive wait for the partner, he smiles, laughs, and confirms that he finds it interesting.

Another aspect of the guiding here in Part (B) is again perceptual economy. When a seeing and hearing child encounter the sound box, we can expect the child to perceive swiftly the box with all the features available from his spatial perspective. Kasper's partner shows Kasper the whole outline of the box, thereby giving him a swift access.

Yet another aspect of this guiding is the culturally sensitive part. At some point a seeing and hearing child will have learned to subsume, to determine, the object under 
the concept of 'box'. He will know this is an example of the universal concept (or rule) 'box'. How can the child with CDB learn to perceive the box as a box?

One way of interpreting our first example is that the guide realizes that Kasper perceives the world differently than she does, in the sense that he has different schemas for organizing his perception of objects. Realizing this difference between herself and Kasper, the partner seeks a common understanding. She does this by way of offering him an understanding that attune to the way seeing and hearing people would encounter the box. The whole box first, with the parts located in it. Though she stills attunes to his blindness by using the exploratory procedure of following edges for perceiving the contour of an object (see Heller and Gentaz 2014). This is then a world neither blind nor sighted way of approaching the box.

Rather than insisting that Kasper perceives the box in a particular manner, she gives him an exemplar of how to encounter the box. He does not have to accept this way of understanding the box: When the partner thinks of the way she shows him the box as an exemplar, of how the box can be encountered, there is in this an acceptance that the box can be encountered differently. She is thus committed to the possibility off changing her take on the box in light of Kasper's exemplar take on it. I take it that when Kasper at 00.52 explores on his own the edges in a similar manner to the way the partner showed him, he gives her recognition for her way of perceiving the box. Both Kasper and the partner can now trust that they have common ground for further communicating about the box.

\section{Recording II}

A bathroom sink is half-full of water. On the front side of the sink Kasper is hanging forward facing in harness attached to his mother's chest. Standing on Kasper's right-hand side is his five-year old big brother. Standing on Kasper's left-hand side is a four-year old friend of the brothers. The friend and the big brother are playing in the water with a toothpaste tube and a lunchbox lid. Kasper's mother has her right hand stretched into the water, playing with a glass. When the recording starts the friend holds the lid at the top, and Kasper touches it the bottom with his right arm that he soon moves over to his mother's right arm so that his is lying next to hers, following her arms line, but not stretched into the water. During the recording Kasper will often shortly touch the mother's right arm with his, checking in on what she is doing. The mother's left hand is also stretched into the water, playing with a cup, with Kasper's left arm also lying next to it. He shortly joins her play with the cup, before he moves his left hand over to the lid that the friend is still holding. He touches the friend. Mother responds by saying "Yes, that is...", and the friend says, "get off". Kasper touches the friend's arm and the lid, starting a negotiation 
over the lid, where the friend tells Kasper again to take his arms off, and takes Kasper's arm and moves it away.

This whole situation starts with guiding Kasper into proximity of the play. Crucially the mother orients her and Kasper's bodies so that they jointly face the sink. The two other boys are also facing the same sink, so all four of them are perceptually oriented to the same space. This bodily alignment between the mother and Kasper, and in some ways the two other boys, creates a field Kasper potentially can perceive. Then, however, the perceptual field is quite complex: There are several objects in the water, and the two older boys are playing and arguing about the lid. The situation is also changing constantly with the two other boys moving their hands around, shifting objects etc. For Kasper, obtaining an overview and understanding of what happens in front of him is difficult, close to impossible due to the time-constraints of tactile perception in situations like this. The $\mathrm{CI}$ is also of limited value in a noisy bathroom with terrible acoustics.

The mother here innovatively uses her arms for giving Kasper access to children playing, giving Kasper some of the benefits of sight through their tactile interaction. This is the two-ness of their interaction where they are showing each other possible ways of organizing the perceptual field.

The phenomenologist Hans Jonas described sight in terms of three characteristics:" (1) simultaneity in the presentation of a manifold, (2) neutralization of the causality of sense-affection, (3) distance in the spatial and mental senses" (1954, p. 507. See Gregersen 2020 for further discussion).

First, we start with Jonas' (2) and (3). Kasper's mother gives Kasper a type of freedom. By letting her arms extend beyond Kasper into the water, the mother shows two things at the same time: That this is significant and that's its' safe to touch the water with the objects. When Kasper lets his arms follow the mother's he uses them to gain freedom. The immediate sensory contact is neutralized through the mother's hands. This neutralization gives him both physical and mental freedom, "distance" in Jonas' words, to choose if he wants to engage with what her arms engages. Importantly he chooses not only to engage with what her arms engages with, but also with his friend. Thereby showing us that he can explore on his own and knows this.

Secondly, and Jonas' (1), recall that Michalko experienced that the guide dog extended his tactile sense so that it also became a distance sense. Michalko was in touch with more than what he touched. In this complex situation, Kasper's mother offers him an organization by literarily extending her arms beyond Kasper's into the water. By using two arms, that Kasper can have several contact points with, she also increases the manifold that Kasper is in touch with. Of course, in amount of manifold, this is not 
equivalent to sight, but if the partner acts as a guide in the sense of being oriented to the most important features she can give him much better access than compared to no guiding. She gives him access to more of the manifold, and to the significant manifold. That is, in organizing the manifold of the perceptual field the mother here manifests to Kasper an exemplar judgment about what is the core of the situation for the three sighted persons. Kasper can use this exemplar judgment as a base from which to explore other features. However, her organization gives him the possibility of orienting himself to the significant features of the perceptual field.

Here Kasper uses his right arm as "checking" in on what his mother's right arm is doing, making sure that since her arm keeps doing the same the significant features of the perceptual field has not changed. Again, then relying on, or trusting, that the mother's arms is oriented to the significant features. With knowing that the right-hand part of the perceptual field is stable, he can use his left arm for accessing other parts of his perceptual field. His left arm has partly contact with the mother's left arm, then knowing how she is oriented, and his left arm is simultaneously negotiating with his friend about the lid.

We have thus seen that this is a kind of "two in one" interaction where the mother provides an exemplar judgment of how to organize the perceptual field. And Kasper is using that exemplar as an organization, but also something he departs from so that he can perceive what interests him the most, namely the friend and the lid. The exemplar judgment is voiced to Kasper as a way of building a community with four members. There is a movement from the subject (the mother), through a judgment of an object (the organization of the sink as the significant feature), that aims at a common understanding in perception and by that participation and negotiation in the community.

The term "guide" suggests that the partner is the one who is active, but what we see here is that Kasper actively utilizes the guide, and her exemplar judgment. The guide provides enabling conditions that the child uses.

Through his mother's body, Kasper is oriented to the play. The social dimension of perception is here fully on display. One person/body through another person/body can be in touch with yet another person/body and their common activities. In this triangle, the middle person/body is the one that makes the contact possible, thus creating an organization of a scene that allows the two other persons/bodies to interact.

\section{Conclusion}

A child with CDB will need partners for many different purposes: For practical tasks; conversations; mobility; descriptions of the environment and so forth. The child also needs the partner for perceptually accessing the environment. This is partly 
because tactile perception often is time-consuming. Having a partner showing the child an organization of the perceptual field is beneficial for mental and perceptual economy. Off-loading perceptual tasks to the partner lets the child faster and with more energy perceive himself. Another reason is that joint perception with the partner can aid in perceiving constantly shifting situations like children playing. Yet another reason for perceptual guidance is that it can attune the child to the partner's way of perceiving, and thereby provide a cultural understanding of the environment. It will create access to more experiences and increase the readability of the partner and culture at large for the child. In the end that will contribute to the child`s self-understanding.

Getting these benefits, demands that the partner and child are closely bodily aligned, sharing a spatial perspective on the world. Within this perspective, the partner can seek to create community with the child through a kind aesthetic activity of creatively organizing the perceptual field.

\section{References}

Allison, H. (2001). Kant's Theory of Taste. A Reading of the Critique of Aesthetic Judgment. New York: NY. Cambridge University Press.

Arendt, H. (1985). The origins of Totalitarianism. New York: NY. Harcourt, Inc.

Granada Gity Council Official Tourist Information Web Page. 2019. http:// en.granadatur.com/que-visitar. Downloaded 9.10.2019.

Gregersen, A. (2018). "Body with Body: Interacting with Children with Congenital Deafblindness in the Human Niche". Journal of Deafblindstudies on Communication. Vol.4. 67-83. Groningen. University of Groningen Press.

Gregersen, A. (2020). "Are We Getting to All That Matters? The need for perceptual guidance." submitted to Journal of Deafblindstudies on Communication. Groningen. University of Groningen Press.

Heller, M. A. and Gentaz, E. (2014). Psychology of Touch and Blindness. New York: NY. Psychology Press.

Jonas, H. (1954). "The Nobility of Sight: A Study in the Phenomenology of The Senses" Philosophy and Phenomenological Research, Vol.14(4). 507-519

Kant, I. (1987). Critique of Judgment. Trans. By Werner S. Pluhar. Indianapolis: IN. Hackett Publishing Company.

Loader, P. (2008). «Mess With My Dog and You Mess With My Mind" in What Philosophy Can Tell You about Your Dog" Chicago and La Salle: IL. Open Court.

Michalko, R. (1999). The Two in One. Philadelphia: PA. Temple University Press.

Nafstad, A.V. and Rødbroe, I.B. (2007). "Co-creating Communication with Persons with Congenital Deafblindness". PP.17-23. Communication Network Update Series, Number 
8. Nordic Staff Training Centre for Deafblind Services. https://nordicwelfare.org/wpcontent/uploads/2017/10/CNUS_08_web.pdf

Seeman, A. (2019). The Shared World. Perceptual Common Knowledge, Demonstrative Communication, and Social Space. London: England. The MIT Press.

Senju, A., Csibra, G., Johnson, M.H. (2008) "Understanding the referential nature of looking: Infants' preference for object-directed gaze" in Cognition Volume 108, Issue 2. https://doi.org/10.1016/j.cognition.2008.02.009

Sterelny, K. (2010). "Minds: extended or scaffolded?" Phenomenology and the Cognitive Sciences, Vol.9(4). 465-481.

Soliman, T.M., R. Ferguson, M.S. Dexheimer, and A. M. Glenberg. (2015). “Consequences of Joint Action: Entanglement with Your Partner." Journal of Experimental Psychology 144 (4): 873-888.

Souriau, J. and Brede, K.S. (2008). "Language and Congenital Deafblindness". Communication Network Updates Series, No 10, NVC. https://nordicwelfare.org/wpcontent/uploads/2017/10/CNUS10_web.pdf

Trevarthen, C. 2011. "The Generation of Human Meaning: How Shared Experience Grows in Infancy". In Seeman, A. (Ed.) Joint Attention. New Developments in Psychology, Philosophy of Mind, and Social Neuroscience. Cambridge: MA. MIT Press.

\section{Acknowledgements}

I would like to thank Rosemarie Van Den Breemer, Kåre Letrud, Anne V. Nafstad as well as the Normativity research group at Inland Norway University of Applied Sciences.

Anstein Gregersen, Chair of Department and Associate Professor of Philosophy, Inland School of Business and Social Sciences/ Department of Law, Philosophy and International Studies, Inland Norway University of Applied Sciences; e-mail: <anstein.gregersen@inn.no>. 
${ }^{1}$ Language, of course, plays an extensive role for both access and participation in the social world. And since sight and hearing are such efficient senses for language perception it is reasonable that communication and language is a central concern for deafblindstudies. However, CDB affects more than language perception, and I will not address language perception directly here.

${ }^{2}$ Axel Seeman defines joint perception the following way:

JP: Two subjects jointly perceive an object just when, due to their communicative interaction, they locate it relative to their respective positions and thus in social space. (2019, p. 163)

I want to note two things about this definition. Seeman describes the communicative interaction the following way: "Joint perception is an intensely bodily undertaking: it requires its subjects to nudge, point, and otherwise direct each other's gaze so as to achieve demonstrative communication" (2019, p 163). Seeman's explication is based on sight. Seeman's "directing each other's gaze" must in the context of CDB be "directing of each other's tactile exploratory procedure". One more thing related to sight here is that from the perspective of CDB a location of an object relative to child and partner's perspective is not enough for joint perception since the two can easily pick out different manifold of the object and categorize it quite differently. I therefore suggest the following definition of joint perception:

JP: Two subjects jointly perceive an object just when, due to their communicative interaction, they locate it relative to their respective positions and furthermore are aware of each other's exploratory procedures, and thus place the object in a social space.

In my terminology the location of the object relative to their respective positions is about which objects are perceived, and the awareness of exploratory procedures is about how the objects are perceived.

${ }^{3}$ For the sake of brevity, I will in the following use "object" to encompass things that can be perceived, such as material objects, persons and events. 
4 The philosophical oriented reader might be stricken by the resemblance between Michalko's description of his interaction with Smokie and what is known in philosophy as "the extended mind". For such a take on Michalko see Loader (2008). My own take on this issue is that the notion of guiding proposed by Michalko and my account of it for children with CDB is better captured with a position called "the scaffolded mind". The scaffolded mind theory of Kim Sterenly (2010) does not deny that there are examples that can be characterized with the extended mind thesis (like Michalko does with his discussion of the long cane. See Michalko 1999, p.117), but that a more salient feature of the human mind is that it is scaffolded through the niche it exists in. Michalko is very sensitive to the fact that his interactions with guide dogs are shaped by society, as he says:

The form a guide will take depends on how blindness is conceived. The first response to blindness is always curative. Can the blind person's sight be restored? If not, what can substitute for sight? How can she move through the world now that she is missing sight? The form of a blind person's social adventure depends on how these questions are answered. How we answer them shapes our conception of blindness and our relation to it, it defines our social relation with blindness. How we conceive of and relate to blindness reveals as Karatheodoris (1982) says, an answer to the question, "What is blindness?" All interactions with blindness simultaneously poses and answers this question. A blind person's search for a guide, then, is always conducted in a social order that understands eyesight as the "natural guide"; as such it becomes a search for something that will replace nature with something "person-created." This made-in-society guide is always "second-best" to the natural one, always "unnatural" in relation to the natural gift of vision. Made in society, made by people, it is techne. (S. 21)

The extended mind theory is about individuals extending their mind through certain items. Michalko's story is about how society, himself and Smokie together constitutes a new world, wherein Michalko's tactile sense is enhanced. The overall picture Michalko draws is about his need for scaffolds, and how we should understand the scaffolds. His proposal is not simply about extending his mind through Smokie.

5 I here select out certain elements of Kant's aesthetic theory that is useful for a model of perceptual guidance. Kant's theory is, of course, much broader and not all of it is relevant for my purposes. I do not think that much hinges on strengths and weaknesses of Kant's larger theory. So, my claims will have to be evaluated on their own and not in relation to that overall theory. 
${ }^{6}$ I here use "proposition" as meaning "A statement or assertion that expresses a judgement or opinion" see: https://en.oxforddictionaries.com/definition/proposition

\section{https://en.oxforddictionaries.com/definition/proposition}

${ }^{7}$ Actually, more correctly, as Henry Allison has pointed out there are three subjects of determination: the concept, the intuition (or perception as we would say today), and the object. See Henry Allison 2001, p. 18.

${ }^{8}$ Thanks to Gøran Forsgren for video footage. 